\title{
Vitamin D, Calcium, Alkaline Phosphatase, and Parathyroid Hormone in Patients with and without Chronic Periodontitis
}

\author{
Dr. Shaili Pradhan, ${ }^{1}$ Dr. Shweta Agrawal, ${ }^{2}$ Dr. Benju Shrestha ${ }^{1}$ \\ ${ }^{1}$ Periodontology and Oral Implantology Unit, Department of Dental Surgery, \\ National Academy of Medical Sciences, Bir Hospital, Kathmandu, Nepal; \\ ${ }^{2}$ Department of Dental Surgery, Itahari Hospital, Sunsari, Nepal.
}

\begin{abstract}
Introduction: Periodontal diseases comprise a group of inflammatory diseases caused by bacteria that colonise the tooth surface and infect surrounding soft tissues, ultimately leading to tooth loss. Vitamin D, calcium, alkaline phosphatase, and parathyroid hormone have role in bone metabolism both in health or in disease.

Objective: To compare serum Vitamin D, calcium, alkaline phosphatase, and parathyroid hormone levels in patients with generalised chronic periodontitis and healthy periodontium.

Methods: A cross-sectional analytical study was done in 80 patients visiting Department of Dental Surgery from July to December 2019 Clinical parameters measured were plaque index, gingival index, clinical attachment level, and pocket depth in the healthy periodontium and generalised chronic periodontitis. Blood investigation for serum Vitamin D, calcium, alkaline phosphatase (ALP), and parathyroid hormone (PTH) level were done. Data was analysed in SPSS v.16.

Results: Out of total 80 patients enrolled (mean age of $36.20 \pm 7.37$ years), 40 (50\%) patients had healthy periodontium and 40 (50\%) had generalised chronic periodontitis. Mean levels of serum Vitamin $\mathrm{D}(\mathrm{P}=0.006)$ and calcium $(\mathrm{P}=0.020)$ were significantly higher in chronic periodontitis patient compared to healthy controls while no significant intergroup differences were seen with respect to ALP (P=0.267) and PTH $(\mathrm{P}=0.351)$.

Conclusion: No significant differences were found in the levels of ALP and PTH between generalised chronic periodontitis and healthy patients and higher levels of Vitamin D and calcium were seen in generalised chronic periodontitis patients as compared to healthy patients.

Keywords: Alkaline phosphatase; bone metabolism; calcium; clinical attachment level; parathyroid hormone; vitamin D.
\end{abstract}

\section{INTRODUCTION}

Periodontal diseases are inflammatory diseases initiated by bacteria that colonise teeth and infect their surrounding soft tissues. Tooth loss can occur due to extension of the inflammation from marginal gingiva to periodontal tissues causing destruction of alveolar bone. ${ }^{1}$

Vitamin D has role in bone metabolism including antiinflammatory activity. ${ }^{2}$ Vitamin D directly induces expression of endogenous antimicrobial peptide cathelicidin, triggered by toll-like receptors in response to bacterial infection. ${ }^{3}$

\section{Correspondence:}

Prof. Dr. Shaili Pradhan

Periodontology and Oral Implantology Unit, Department of Dental Surgery, National Academy of Medical Sciences, Bir Hospital, Kathmandu, Nepal.

email: shaili_p@yahoo.com

\section{Citation}

Pradhan S, Agrawal S, Shrestha B. Vitamin D, Calcium, Alkaline Phosphatase, and Parathyroid Hormone in Patients with and without Chronic Periodontitis. J Nepal Soc Perio Oral Implantol. 2020 Jul-Dec;4(8):51-6.
Calcium deficiency results in bone loss and increased inflammation which are specific characteristic features of periodontitis. ${ }^{4}$ Alkaline phosphatase (ALP) is hydrolase enzyme produced by various cells, within periodontium and gingival crevice. It allows bone mineralisation by releasing organic phosphate and hydrolysing inorganic pyrophosphate. ${ }^{5}$ Parathyroid hormone (PTH) exerts both anabolic and catabolic action on bone. ${ }^{6}$ They are important for the bone metabolism and may be associated with the alveolar bone loss.

Sixty-five percent of cross-sectional studies included in systematic review have reported significant association between low Vitamin D levels and poor periodontal parameters. ${ }^{7}$ The prevalence of periodontitis in Nepalese populations has been shown higher. ${ }^{8}$ The aim of this study was to compare serum Vitamin D, calcium, alkaline phosphatase, and parathyroid hormone levels in patients with healthy periodontium and generalised chronic periodontitis.

\section{METHODS}

An analytical cross-sectional study was conducted in patients with healthy periodontium and chronic 
periodontitis attending Department of Dental Surgery, Bir hospital, Kathmandu, Nepal from July 2019 till December 2019. Ethical approval was obtained from Institutional Review Board, Ethical Committee of National Academy of Medical Sciences, Bir Hospital, Kathmandu, Nepal (NAMSIRB 877/076/077). Informed consent was obtained from patients.

Sample size was determined taking standard deviation value of 0.8 (for Vitamin D level) from study by Dietrich et al. $2004 .^{9}$ Power analysis was done to determine the sample size. As difference between mean was taken as 0.5 (Cohen's effect) with standard deviation of 0.8 ; 7 error (5\%); power (1- 1 ) $80 \%$, hence, minimum sample size required was 40 per group (total of 80 patients). The data was collected by convenience sampling method. The clinical examination was performed and recorded by single examiner (Agrawal S). Periodontal parameters measured were plaque index, ${ }^{10}$ gingival index, ${ }^{11}$ clinical attachment level, and pocket depth in order to decide presence of healthy periodontium and generalised chronic periodontitis. Patients with loss of attachment at more than $30 \%$ sites were diagnosed as generalised chronic periodontitis. After baseline measurements, patients were sent for blood investigations for serum Vitamin D, Calcium, PTH and ALP levels. All investigations were performed in Biochemistry laboratory, Bir hospital. Chemiluminescence Immunoassay (CLIA) machine was used for estimation of Vitamin D and PTH levels.

Principle of serum ALP level determination: ${ }^{12}$ At alkaline $\mathrm{pH}$, ALP present in the sample catalyses the reaction where the substrate (p-nitrophenyl phosphate) is converted into 4-nitrophenol that has intense yellow colour. The reaction is monitored by measuring the rate of increase in absorbance by auto analyser at 405 or $415 \mathrm{~nm}$ which is proportional to the activity of ALP in the serum.

Principle of serum calcium level determination: ${ }^{13}$ At $\mathrm{pH}$ of 7.8, Arsenazo III combines with calcium ions in the sample to form coloured chromophore, the absorbance of which is measured at $650 \mathrm{~nm}$ and is proportional to calcium concentration.

Principle of chemiluminescent immunoassay: ${ }^{14}$ Antigen molecule (such as peptides, hormones, and proteins) are identified in the sample by using enzyme labelled antibodies. The enzyme used converts substrate used to a reaction product, which emits light instead of developing particular colour. The amount of particular biological molecule in the sample is based on luminescence observed which is determined by CLIA machine. The advantage of chemiluminescence over absorbance in that the former is an absolute measure whereas the latter is relative.

The inclusion criteria of participants were: age 25-54 years with generalised chronic periodontitis and healthy periodontium attending Department of Dental Surgery, Bir Hospital. The reason for not including older population is due to exclusion of menopausal women from the study and including higher age groups would involve enrollment of only male subjects. Patient with generalised clinical attachment loss of $3 \mathrm{~mm}$ to $\geq 6 \mathrm{~mm}$ were included in chronic periodontitis group while patient with normal probing depth of 2-3 mm were included in healthy periodontium group. Exclusion criteria were patients with systemic disease that are known to affect the periodontium, immuno-compromised patients, bone disorders, metabolic disorders, menopausal women, pregnancy and lactating female, uncooperative patient, patient who is not willing to participate, and does not give informed consent.

All participants who met inclusion criteria were examined to measure periodontal pocket depth, clinical attachment level in six sites per tooth using University of North Carolina-15 periodontal probe. Gingival index and plaque index were measured at four sites per tooth to diffierentiate healthy and chronic periodontitis patients.

The data were entered in Microsoft Excel sheet and analysed using SPSS Statistics for Windows, version 16.0 (SPSS Inc., Chicago, Ill., USA) software and independent t-test was used for intergroup comparison of mean levels of Vitamin D, calcium, ALP, and PTH as well as for genderwise comparison. ANOVA was used to compare means of Vitamin D, calcium, ALP, and PTH levels between different age groups.

\section{RESULTS}

Total of 80 patients were enrolled in the study (40,50\% patients with healthy periodontium and 40,50\% patients with generalised chronic periodontitis). Forty were female (23, 57.5\% healthy and 17, 42.5\% with generalised chronic periodontitis) and 40 were male (17, $42.5 \%$ healthy and 23 , $57.5 \%$ with generalised chronic periodontitis) with mean age of $36.20 \pm 7.37$ years.

The overall mean values of Vitamin D, Calcium, ALP, and PTH levels were $19.44 \pm 6.03 \mathrm{ng} / \mathrm{mL}, 8.65 \pm 0.59 \mathrm{mg} / \mathrm{dL}, 75.61 \pm 21.27$ $\mathrm{U} / \mathrm{L}$ and $43.56 \pm 22.52 \mathrm{pg} / \mathrm{mL}$ respectively. The mean Vitamin D, Calcium, ALP, and PTH levels in male and female are shown in Table 1. Mean levels of Vitamin D ( $\mathrm{P}=0.123)$, Calcium ( $\mathrm{P}=0.227)$, ALP $(\mathrm{P}=0.671)$, and $\mathrm{PTH}(\mathrm{P}=0.460)$ were statistically not significant on genderwise comparison.

The mean Vitamin D, Calcium, ALP, and PTH level in patient with healthy periodontium and with generalised chronic periodontitis are shown in Table 2. Mean levels of Vitamin D $(\mathrm{P}=0.006)$ and Calcium $(\mathrm{P}=0.020)$ were statistically significant with higher level in chronic periodontitis group. ALP $(\mathrm{P}=0.267)$ and $\mathrm{PTH}(\mathrm{P}=0.351)$ were statistically not significant between patients with healthy periodontium and chronic periodontitis. 
Table 1: Mean vitamin D, calcium, ALP, and PTH levels in male and female.

\begin{tabular}{|l|l|c|c|}
\hline \multicolumn{1}{|c|}{ Serum markers } & \multicolumn{1}{|c|}{ Gender } & Mean \pm SD & P value \\
\hline Vitamin D (ng/mL) & Female & $18.47 \pm 6.22$ & 0.123 \\
\hline Calcium $(\mathrm{mg} / \mathrm{dL})$ & Male & $20.55 \pm 5.70$ & 0.227 \\
\hline Alkaline Phosphatase $(\mathrm{U} / \mathrm{L})$ & Female & $8.58 \pm 0.63$ & 0.671 \\
\hline & Male & $8.74 \pm 0.55$ & 0.460 \\
\hline Parathyroid hormone $(\mathrm{pg} / \mathrm{mL})$ & Fale & $74.65 \pm 22.99$ & $76.68 \pm 19.37$ \\
\hline
\end{tabular}

Table 2: Mean values of vitamin D, calcium, ALP, and PTH levels in patients with healthy periodontium and chronic periodontitis.

\begin{tabular}{|c|c|c|c|}
\hline Serum markers & Test/Control & Mean \pm SD & $P$ value \\
\hline \multirow{2}{*}{ Vitamin D (ng/mL) } & Healthy periodontium & $17.69 \pm 5.44$ & \multirow{2}{*}{0.006} \\
\hline & Generalised chronic periodontitis & $21.33 \pm 6.08$ & \\
\hline \multirow{2}{*}{ Calcium (mg/dL) } & Healthy periodontium & $8.51 \pm 0.55$ & \multirow{2}{*}{0.020} \\
\hline & Generalised chronic periodontitis & $8.81 \pm 0.60$ & \\
\hline \multirow{2}{*}{ Alkaline Phosphatase (U/L) } & Healthy periodontium & $73.03 \pm 20.25$ & \multirow{2}{*}{0.267} \\
\hline & Generalised chronic periodontitis & $78.30 \pm 21.94$ & \\
\hline \multirow{2}{*}{ Parathyroid hormone (pg/mL) } & Healthy periodontium & $41.66 \pm 24.77$ & \multirow{2}{*}{0.351} \\
\hline & Generalised chronic periodontitis & $46.44 \pm 20.68$ & \\
\hline
\end{tabular}

Table 3: Level of vitamin D, Calcium, ALP and PTH according to age groups.

\begin{tabular}{|c|c|c|c|c|}
\hline Serum markers & Age groups & n (\%) & Mean \pm SD & $P$ value \\
\hline \multirow{4}{*}{ Vitamin D (ng/mL) } & 24-33 years & 35 (43.75) & $17.71 \pm 5.03$ & \multirow{4}{*}{0.030} \\
\hline & 34-43 years & $30(37.5)$ & $19.88 \pm 6.50$ & \\
\hline & $44-54$ years & 15 (18.75) & $22.49 \pm 6.12$ & \\
\hline & Total & $80(100)$ & $19.44 \pm 6.03$ & \\
\hline \multirow{4}{*}{ Calcium (mg/dL) } & 24-33 years & $35(43.75)$ & $8.60 \pm 0.51$ & \multirow{4}{*}{0.261} \\
\hline & $34-43$ years & $30(37.5)$ & $8.79 \pm 0.65$ & \\
\hline & $44-54$ years & $15(18.75)$ & $8.50 \pm 0.64$ & \\
\hline & Total & $80(100)$ & $8.65 \pm 0.59$ & \\
\hline \multirow{4}{*}{ Alkaline Phosphatase (U/L) } & 24-33 years & 35 (43.75) & $75.32 \pm 22.75$ & \multirow{4}{*}{0.977} \\
\hline & 34-43 years & $30(37.5)$ & $75.40 \pm 20.44$ & \\
\hline & $44-54$ years & $15(18.75)$ & $76.67 \pm 20.86$ & \\
\hline & Total & $80(100)$ & $75.61 \pm 21.27$ & \\
\hline \multirow{4}{*}{ Parathyroid hormone (pg/mL) } & 24-33 years & $35(43.75)$ & $49.69 \pm 25.42$ & \multirow{4}{*}{0.085} \\
\hline & $34-43$ years & $30(37.5)$ & $37.31 \pm 20.07$ & \\
\hline & $44-54$ years & $15(18.75)$ & $42.16 \pm 17.03$ & \\
\hline & Total & $80(100)$ & $43.56 \pm 22.52$ & \\
\hline
\end{tabular}

Mean levels of Vitamin D, Calcium, ALP, and PTH in different age groups are shown in Table 3. Mean level of Vitamin D $(\mathrm{P}=0.030)$ was statistically significant among different age groups, 24-33 years age group showing lower values than others. Calcium ( $\mathrm{P}=0.261)$, ALP $(\mathrm{P}=0.977)$, and PTH $(\mathrm{P}=0.085)$ were statistically not significant when compared between different age groups.

\section{DISCUSSION}

The present study showed no significant differences in the levels of PTH and ALP among healthy and generalised chronic periodontitis groups. However, higher levels of Vitamin D and Calcium were found in generalised chronic periodontitis group compared to healthy controls which 
showed that deficiency of Vitamin D is not associated with periodontal diseases.

Vitamin D not only has effect on bone mineral density but also is said to have immunomodulatory effects that might alter the course of periodontal disease. ${ }^{9}$ Vitamin D is essential for calcium absorption and thereby maintains serum calcium levels favouring bone deposition and maintaining bone mineral density. Reduced bone mineral density is a known risk factor for periodontal disease. Dietrich et al. ${ }^{9}$ found Vitamin D3 concentrations to be inversely related to the loss of attachment in population greater than or equal to 50 years of age. However, in population (both men and women) less than 50 years, no significant association was found between Vitamin D3 and loss of attachment. The current study also shows no relation of Vitamin D levels with periodontal health and disease. This might be due to less inclusion of patients above 50 years of age. Besides, the inverse relation seen in patient above 50 years in study by Dietrich et al. might be due to increased prevalence and extent of attachment loss in older individuals relative to the young individuals.

The other study done by Dietrich et al. who took data from the third National Health and Nutrition Examination Survey (NHANES III), analysed it and reported that the anti-inflammatory effects of Vitamin D might be helpful in reducing susceptibility to gingival inflammation. ${ }^{15}$ However, plaque levels were not assessed in NHANES III which is an important confounder variable. Furthermore, considering their cross-sectional study design, the causeeffect relationship could not be established.

A systematic review ${ }^{16}$ concluded that the association between serum Vitamin D levels and periodontal disease in older adults is not significant which supports the findings of the present study. The present study, in fact, showed higher Vitamin D and calcium levels in chronic periodontitis patients. In contrast to the present study where Vitamin D was higher in periodontitis group, Laky et al. had shown positive association of deficit Vitamin D levels with onset and progression of periodontal disease. ${ }^{17}$ There was also a systematic review that reported inconclusive data to support the association between serum Vitamin D levels and periodontal disease. ${ }^{7}$

Similar to the findings of current study, Zhang et al. ${ }^{18}$ found increased level of serum Vitamin D in generalised aggressive periodontitis patient. The increased level of serum Vitamin D was attributed to increased plasma concentration of Vitamin D binding protein. ${ }^{18}$ Zhang et al. also found increase in serum IL-6 level and neutrophil count and stated that increased levels of neutrophils and IL-6 in periodontitis patient might contribute to increased concentrations of Vitamin D binding protein in plasma thereby increasing serum Vitamin D levels.
A study in the third National Health and Nutrition Examination Survey (NHANES III), reported that low dietary intake of calcium was associated with periodontal disease in young males and females and in older males. ${ }^{19}$ They found increased severity of periodontal disease in subjects having less dietary intake of calcium. As per this finding, the calcium levels is supposed to be reduced in patient with increased attachment loss but, the present study showed high serum calcium levels in chronic periodontitis patients. This increased value might be attributed to the ongoing bone resorption that increases serum calcium as a compensatory mechanism. Besides, dietary calcium was not evaluated in the present study.

Vitamin D, in addition to its role in maintaining serum calcium level and bone homeostasis, is also said to act as an anti-inflammatory agent by inhibition of cytokine expression by immune cells. It is also said to cause secretion of antimicrobial factors by monocyte/macrophages. ${ }^{20}$ Hence, Garcia et al. ${ }^{20}$ administered supplements of Vitamin D (>400 IU/day) and calcium ( $>1000 \mathrm{mg} /$ day) in periodontitis patient under maintenance therapy and with residual pockets at two or more sites and found improved all over clinical parameters over 12 months and concluded that calcium and Vitamin D supplementation had a positive effect on periodontal health. On contrary to this, the present study showed higher levels of calcium and Vitamin D in the chronic periodontitis compared to healthy group and the overall mean Vitamin D level was also lesser than normal level. However, no Vitamin D and calcium supplements were given in the current study. Besides, serum Vitamin D levels may not be solely dependent on the dietary intake of Vitamin D and even the exposure to ultraviolet rays from sun is important determinant of serum Vitamin D concentrations. In the study by Garcia et al., in addition to calcium and Vitamin supplementation, the patients were maintained on regular follow-up where deplaquing was performed. ${ }^{20}$ Hence, this might also have affected their outcomes.

Calcium deficiency is reported to be associated with osteopenia and leads to resorption of alveolar bone of rats which is in contrast to the present study which showed higher level of calcium in chronic periodontitis group compared to the healthy group. ${ }^{21}$ Periodontitis patients exhibited a significant increase in serum calcium levels which may be due to the negative calcium balance mechanism to maintain the homeostasis. The secretion of parathyroid hormone removes calcium from bone including alveolar bone, which in turn, leads to increase in serum calcium levels.

Uhrbom et al. reported calcium supplementation does not influence the periodontal status of patients with periodontitis.5 Puspa rani et al. had reported significant increase in serum calcium and ALP level in patients with 
diabetes and periodontitis, which supports the present study, however, in the present study diabetes patients were excluded. ${ }^{22}$ Mahalingam et al. reported increase in serum calcium level in periodontitis and its progression which is similar to the present study. However, the limitation in the present study was that severity of periodontitis was not evaluated. ${ }^{4}$

Parathyroid hormone is one of the important regulators of calcium homeostasis. It is produced by parathyroid glands when serum calcium level drops below normal and it increases serum calcium level by increasing calcium absorption from intestine, reducing calcium excretion from kidneys and by increasing osteoclastic bone resorption. Hence, PTH has both catabolic and anabolic effects on bone metabolism. ${ }^{6}$ It causes bone resorption as well as it increases calcium absorption that favours bone deposition. The net effect depends on the duration of exposure to the PTH. Continuous exposure to the PTH causes shift in polarity towards the resorptive processes in bone while intermittent exposure is said to cause bone apposition in maxilla and mandible. ${ }^{6}$ Antonoglou et al. had reported no association of serum PTH and periodontitis showing no significant differences between healthy and periodontitis groups and smoker and non-smoker, which supports the present study which showed non-significant result compared in the both groups. ${ }^{23}$ Both animal ${ }^{24,25}$ and human ${ }^{26}$ experiments have shown that following intermittent administration of PTH or its recombinant, there was reduction in alveolar bone resorption, improved bone healing and inflammatory cells number were also found to be reduced in areas of gingival inflammation. However, in the present study, PTH was not administered.

ALP is an essential enzyme as it has important role in normal turnover process of periodontium. It is released from polymorphonuclear neutrophils during inflammation, osteoblast during bone formation and periodontal ligament fibroblast during periodontal regeneration. ${ }^{27}$ Caula et al. showed significant correlation between alkaline phosphatase and periodontitis which showed higher serum alkaline phosphatase levels. ${ }^{27}$ Gibert et al. showed significantly lower bone-type ALP activity in patients with greater attachment loss than that in control patients. However, there was no significant difference in total ALP activity. ${ }^{28}$ Pradhan et al. found significantly increased salivary as well as serum ALP levels in generalised chronic periodontitis patients and after two months of non-surgical periodontal therapy significant reduction was seen in salivary ALP levels; however, no significant reduction was seen in serum ALP levels after two months. ${ }^{29}$ This might have occurred as serum ALP level depends on function of other organs as well such as bone, kidney, liver, etc. The present study showed no association between chronic periodontitis and serum ALP levels. Serum levels of ALP were slightly higher in chronic periodontitis patient than healthy controls but not statistically significant and the severity of periodontitis was also not assessed in current study. Nevertheless, reduction in ALP levels were seen after non-surgical periodontal therapy in chronic periodontitis patients.

Schulze-Spate et al. had evaluated the association between serum bone metabolism biomarkers (calcium, Vitamin D, PTH, ALP, phosphate) with onset and progression of periodontal disease in older males and reported that Vitamin D and PTH were associated with severe periodontal diseases, with higher PTH and lower Vitamin D. However, they included men of $>65$ years in the study and higher prevalence and extent of attachment loss is seen in older subjects than in younger subjects. ${ }^{30}$

The limitations of the present study include small sample size, severity of chronic periodontitis was not compared with Vitamin D, calcium, alkaline phosphatase, and parathyroid hormone level. The age distribution between two groups was not similar as chronic periodontitis group had more old aged patients compared to healthy group; however, they were within the inclusion criteria for age group.

\section{CONCLUSION}

Within the limitation of this study, it is concluded that there are no differences in the level of ALP and PTH between generalised chronic periodontitis and healthy patients and higher level of Vitamin D and calcium were seen in generalised chronic periodontitis patients as compared to healthy patients. No association was seen between changes in the level of Vitamin D, Calcium, ALP, and PTH and generalised chronic periodontitis.

Further study is required to compare serum Vitamin D, calcium, alkaline phosphatase and parathyroid hormone level in generalised chronic periodontitis and healthy patients and its association.

\section{ACKNOWLEDGEMENT}

This research was funded by Research and Grant Committee of National Academy of Medical Sciences.

Conflict of Interest: None. 


\section{REFERENCES}

1. Petersen PE, Baehni PC. Periodontal health and global public health. Periodontol 2000. 2012;60:7-14.

2. do Amaral Bastos J, de Andrade LCF, Ferreira AP, de Almeida Barroso E, de Castro Daibert P, de Sa Barreto PL, et al. Serum levels of vitamin D and chronic periodontitis in patients with chronic kidney disease. J Bras Nefrol. 2013;35(1):20-6.

3. Van der Velden U, Kuzmanova D, Chapple ILC. Micronutritional approaches to periodontal therapy. J Clin Periodontol. 2011;38(Suppl 11):142-58.

4. Mahalingam A, Ilango P, Suresh V, Perumal K, Kathiresan H. Evaluation of serum calcium levels in patients with periodontitis. Int J Curr Adv Res. 2017 Feb;6(2):2287-9.

5. Urhbom E, Jacobson L. Calcium and periodontitis: Clinical effect of calcium medication. J Clin Periodontol. 1984;11:230-41.

6. Abuduwali N, Lossdorfer S, Winter J, Kraus D, Guhlke S, Wolf M, et al. Functional characterization of the parathyroid hormone 1 receptor in human periodontal ligament cells. Clin Oral Invest. 2014;18(2):461-70.

7. Pinto JP, Goergen J, Muniz FW, Haas AN. Vitamin D levels and risk for periodontal disease: A systematic review. J Periodontal Res. 2018;53(3):298-305.

8. Pradhan S, Bhatta MK. Assessment of periodontal status of rural Nepalese population using the community periodontal index. J Nepal Dent Assoc. 2009;10(2):97-104.

9. Dietrich T, Joshipura KJ, Dawson-Hughes B, Bischoff-Ferrari HA. Association between serum concentrations of 25-hydroxyvitamin D3 and periodontal disease in the US population. Am J Clin Nutr. 2004;80:108-13.

10. Loe H. The gingival index, the plaque index and the retention index systems. J Periodontol. 1967;38(6):38-44.

11. Löe H, Silness J. Periodontal disease in pregnancy I. Prevalence and severity. Acta Odontol Scand. 1963;21(6):533-51.

12. Breslow R, Katz I. Relative reactivities of p-nitrophenyl phosphate and phosphorothioate toward alkaline phosphatase and in aqueous hydrolysis. J Am Chem Soc. 1968;90(26):7376-7.

13. Leary NO, Pembroke A, Duggan PF. Single stable reagent (Arsenazo III) for optically robust measurement of calcium in serum and plasma. Clin Chem. 1992 Jun 1;38(6):904-8.

14. Cinquanta L, Fontana DE, Bizzaro N. Chemiluminescent immunoassay technology: what does it change in autoantibody detection? Auto Immun Highlights. 2017 Dec 1;8(1):9-16.

15. Dietrich T, Nunn M, Dawson-Hughes B, Bischoff-Ferrari H. Association between serum concentrations of 25-hydroxyvitamin D and gingival inflammation. Am J Clin Nutr. 2005;82:575-80.

16. Van der Putten GJ, Vanobbergen J, Visschere LD, Schols J, deBaat C. Association of some specific nutrient deficiencies with periodontal disease in elderly people: A systematic literature review. Nutrition. 2009;25:717-22.

17. Laky M, Bertl K, Haririan H, Andrukhov O, Seeman R, Volf I, et al. Serum levels of 25-hydroxyvitamin D are associated with periodontal disease. Clin Oral Investig. 2016;21:1553-8.

18. Zhang X, Meng H, Sun X, Xu L, Zhang L, Shi D, et al. Elevation of vitamin D-binding protein levels in the plasma of patients with generalized aggressive periodontitis. J Periodontal Res. 2013 Feb;48(1):74-9.

19. Nishida M, Grossi SG, Dunford RG, Ho AW, Trevisan M, Genco RJ. Calcium and the risk for periodontal disease. J Periodontol. 2000;71:1057-66.

20. Garcia MN, Hildebolt CF, Miley D, Dixon D, Coutture RA, Anderson-Spearie CL, et al. One-year effects of vitamin D and calcium supplementation on chronic periodontitis. J Periodontol. 2011;82:25-32.

21. Lundgren S, Rosenquist JB. Short term bone healing in calcium deficiency osteopenia and disuse osteopenia: experimental studies in adult rats. Scand J Dent Res. 1992;100(6):337-9.

22. Pushpa Rani DS, Anandan SM. A clinical study of serum alkaline phosphatase and calcium level in type 2 diabetes mellitus with periodontitis among the south Indian population. SRM J Res Dent Sci. 2012;3:175-9.

23. Antonoglou GN, Knuuttila M, Niemelea O, Yleostalo P, Raunio T, Hiltunen L, et al. Serum parathyroid hormone and active vitamin D in chronic periodontitis. J Clin Periodontol. 2015;42:726-32.

24. Barros SP, Silva MA, Somerman MJ, Nociti FH. Parathyroid hormone protects against periodontitis-associated bone loss. J Dent Res. 2003;82:791-5.

25. Vasconcelos DF, Marques MR, Benatti BB, Barros SP, Nociti FH, Novaes PD. Intermittent parathyroid hormone administration improves periodontal healing in rats. J Periodontol. 2014;85:721-8.

26. Bashutski JD, Eber RM, Kinney JS, Benavides E, Maitra S, Braun TM, et al. Teriparatide and osseous regeneration in the oral cavity. New Engl J Med. 2010;363(25):2396-405.

27. Caula AL, Lira-Junior R, Tinoco EMB, Fischer RG. Serum creatinine and alkaline phosphatase levels are associated with severe chronic periodontitis. J Periodontol Res. 2015;50:793-7.

28. Gibert P, Tramini P, Sieso V, Piva MT. Alkaline phosphatase isozyme activity in serum from patients with chronic periodontitis. J Periodontol Res. 2003;38:362-5.

29. Pradhan S, Koirala PK. Alkaline phosphatase levels before and after nonsurgical periodontal therapy. J Nepal Soc Perio Oral Implantol. 2019;3(6):62-5.

30. Schulze-Spate U, Turner R, Wang Y, Chao RP, Schulze PC, Phipps K, et al. Relationship of bone metabolism biomarkers and periodontal disease: The osteoporotic fractures in men (MrOS) study. J Clin Endocrinol Metab. 2015;100(6):2425-33. 